\title{
Boundaries, gaps, and overlaps: defining roles in a multidisciplinary nephrology clinic
}

\author{
This article was published in the following Dove Press journal: \\ Journal of Multidisciplinary Healthcare \\ 9 October 2014 \\ Number of times this article has been viewed
}

\author{
Terese Stenfors-Hayes' \\ Helen $\mathrm{H}_{\text {Kang }}^{2}$ \\ 'Department of Learning, Informatics, \\ Management and Ethics, Karolinska \\ Institutet, Stockholm, Sweden; ${ }^{2} B C$ \\ Centre for Excellence in HIVIAIDS, \\ Vancouver, BC, Canada
}

\begin{abstract}
This study aims to explore how health care professionals in a multidisciplinary chronic kidney disease clinic interact with one another, patients, families, and caregivers to expand understanding of how this increasingly common form of chronic disease management functions in situ. Nonparticipatory observations were conducted of 64 consultations between patients and health care professionals and end-of-day rounds at a multidisciplinary chronic kidney disease clinic. Key themes in our findings revolved around the question of boundaries between the health professions that were expected to work cooperatively within the clinic, between medical specialties in the management of complex patients, and between caregivers and patients. Understanding the importance of various professional roles and how they are allocated, either formally as part of care design or organically as a clinical routine, may help us understand how multidisciplinary care teams function in real life and help us identify gaps in practice. This study highlights two areas for further study and reflection: the effect of discrepancies in health information and the role of caregivers in patient care.
\end{abstract}

Keywords: clinical medicine, interprofessional relations, observation, qualitative research

\section{Introduction}

Chronic kidney disease (CKD) is included among the "complex diseases" that can benefit from a multidisciplinary approach. Such an approach can be seen in clinics in which physicians, nurses, pharmacists, social workers, and other health professionals provide integrated care for patients from their initial diagnosis. ${ }^{1}$ Canadian nephrologists are strongly supportive of multidisciplinary clinics (MDCs), ${ }^{2}$ which, with other complex diseases, have been shown to contribute to improved patient outcomes, greater job satisfaction for health care professionals (HCPs), and costsavings for governments. ${ }^{3-7}$ However, such clinics have proved difficult to sustain, ${ }^{8}$ and little is known about their actual implementation and development. Models of care for CKD patients have not been subjected to the same scrutiny as pharmacologic interventions, and therefore there is little basis for developing best practice guidelines for clinic structure, nor for comparative understanding of resource allocation costs. ${ }^{9}$

Multidisciplinary, interprofessional, or interdisciplinary care ${ }^{10}$ generally refer to the degree of collaboration and shared decision-making ${ }^{11,12}$ in or across a clinical setting. This may range from occasional interactions among the HCPs to structured and regular meetings and consultations. The use of this loose cluster of models in chronic care is increasing, but little is known about what happens in MDC settings in practice. Studies in this field tend to be based on interview data, rather than capturing the work in situ, ${ }^{13}$
Correspondence: Terese Stenfors-Hayes Department of Learning, Informatics, Management and Ethics, Karolinska Institutet, 17177 Stockholm, Sweden Tel +46852483737

Fax +468311101

Email terese.stenfors-hayes@ki.se 
and focus primarily on the HCPs without recognizing the role of the patient, nor that of their family or caregiver who, in the MDC model, often function as lay experts. ${ }^{8}$

In this study, we observed the day-to-day activities of one MDC, focusing on how HCPs interacted with one another, patients, families and caregivers to understand how clinical relationships emerge in this setting. Our aim was to expand our understanding of how this increasingly common form of chronic disease management functions in situ.

\section{Analytical framework}

Our study is framed through Bourdieu's relational approach to social interactions within their broader social structures. Bourdieu proposed two terms to account for the structural opportunities and constraints and to account for how individuals negotiate these options. The term field describes spaces in which people with different kinds of assets or stakes struggle to position themselves to maintain or improve their relative position. These assets may be cultural (eg, competency in medical language), social (eg, networks of friends and family), and symbolic (eg, honor and prestige). ${ }^{14}$ The term habitus refers to nonintellectualized "dispositions"; that is, bodily and perceptual systems that individuals develop as a result of their immersion in a field. The disposition of the habitus is tied to a group (eg, kidney patients or nurses) and is by definition shaped by the forces of a field. ${ }^{15}$ This differs from social interaction theory by more heavily weighing the structural impact of the field rather than individualized interactions. In this way, the habitus does not force people to behave in a specific way but is the range of possible actions available to individuals positioned in a particular way. ${ }^{16}$ Hence, people encounter both choices and limitations in a given field and navigate this space of possibilities and constraints with tacit know-how and practical strategies they have developed as a result of a deep immersion in the field. This is a useful way of investigating an MDC because each type of HCP enters the clinic with a habitus developed in their respective professions. It is through that perceptual framework that they make sense of CKD and make practical decisions about care. We hypothesize that it is the tensions and incompatibilities between the habituses of each HCP, patients and their families that make MDCs difficult to implement. To test this hypothesis, we followed Bourdieu's approach to observations ${ }^{17}$ and conceptualized the visits as an interaction between competing habituses that were in the process of developing into a shared habitus.

\section{Methods}

\section{The clinic}

The clinic has approximately 1,200 renal patients in their care, with each patient being assigned a primary nurse. The clinic has in total seven nephrologists, five nurses, four dieticians, one social worker, and one pharmacist. It is open four times a week for 4 hours each time. When patients come to the clinic, they have several consecutive appointments with different HCPs. They always see a nurse and a nephrologist, and sometimes also a dietician, a pharmacist, or a social worker according to the HCPs' assessment of the patient's needs or the patient's own requests. The HCP meets the patient in the waiting area, and they walk together to the consultation room. After each consultation, the HCP walks back with the patient to the waiting area. After each clinic shift, all HCPs attend the end-of-day rounds, which are led by one of the nephrologists.

\section{Data collection}

Between December 2012 and February 2013, we (two trained qualitative researchers) attended 18 different clinic shifts, arriving early to observe the opening of the clinic and staying until after the clinic closed to attend the round. During the first shifts, we focused our observations on nephrologists' visits (consultation) with patients. In the later weeks, we followed patients through all of their appointments at the clinic. We did not ask the patients any questions during the visits with their HCPs, nor did we ask the patients questions between visits. However, between visits, we sometimes asked clarifying questions to the HCP, or the HCP sometimes wanted to explain something to us. In addition, we spent time near the reception area, where most of the interaction between HCPs took place. We also spent time in the waiting area to observe whether any interactions between patients were taking place, which was not the case.

In total, 42 patients were observed through 64 visits with HCPs. We also attended the end-of-day rounds in which the HCPs discussed the cases of the day. The clinic often had visitors and students in attendance, so both staff and patients were used to being observed, which limited the Hawthorne effects in the data collection. Ethical approval for the study was granted by the university and the hospital where the study took place. We took extensive field notes, which we transcribed and shared with one another. When we were at the clinic on the same day, we verbally debriefed after the clinic closed. On days where we were not at the clinic simultaneously, we debriefed by telephone or email. We concluded our observations when a discernible pattern emerged with 
regard to the relational dynamics at the clinic among HCPs, patients, and caregivers.

\section{Analysis}

Data analysis was conducted alongside fieldwork, and as described earlier, fieldwork was adapted accordingly. ${ }^{18}$ We analyzed the field notes using thematic analysis ${ }^{19}$ and conceptualized the visits as interactions between competing habituses that were in the process of developing into a shared habitus. ${ }^{17}$ We read all transcripts several times and then generated initial codes, which we illustrated with extracts from the transcripts. After discussing and reviewing these codes, we grouped them into categories. We then named the categories and wrote a fuller vignette for each. For this publication, we focused on the categories that were related to the broader theme of multidisciplinary care. We then revisited the transcripts with this theme in mind and refined the categories. Classification of categories was independent of the frequency with which they were identified in the transcripts. As well as looking for convergence of themes, we also sought negative instances to ensure the analysis was not too aligned with our emerging notions of the findings. We discussed the categorization until negotiated consensus ${ }^{20}$ was achieved to minimize the influence of researcher subjectivity and preconceptions.

\section{Results}

Key themes in our data revolved around the question of boundaries between the health professions that were expected to work cooperatively within the clinic, between medical specialties in the management of complex patients, and between caregivers and patients.

\section{Walls within walls: professional boundaries}

The heart of the clinic was in front of the daily patient board, which was the central shared mechanism for managing patient flow. The board acted as the communicative anchor for the clinic staff. An important part of the interaction between HCPs took place as the HCPs reviewed the board together. This review also offered a few seconds of breathing space between appointments. Despite having patients sign in and an implicit system of timing of being seen, there was sometimes variability in how and when patients were seen as HCP's "claimed" patients by putting their initials beside them. Sometimes this was based on personal preference (“Oh, I'm gonna put my name down for this guy. He's my favorite. He is adorable!" [nurse]), but more often it happened for continuity of care purposes. The nephrologists and the head nurse "tidied up" the board throughout the day, marking off completed encounters and getting an overview of what remained to be done. When patients were taken ahead of appointment times, other members of the team, not knowing the rationale, on a couple occasions had some negative comments about that. Managing the board allowed a level of control, and in the process, other HCPs were occasionally told whom they should see, which order worked, and which order did not.

Physician: You cannot do this [see patients in a more flexible order]. You have to do this [the way it is supposed to be], or it is not working for us [the physicians]. This is unacceptable. I have been here since X o'clock, and I have not been able to see anyone yet!

The nurses at the clinic had a crucial role in acting as the link between patients and the HPCs, as they always invited the patients to ask lots of questions, sometimes answering these questions themselves and other times directing patients to seek an answer from another HCP: "Any questions for me, or for me to pass on?" or "It's a valid question, talk to the doctor or pharmacist." The nurse could therefore become a gatekeeper or a guide regarding what the patient may focus on as they made their way through their appointments with the various HCPs. So central was the nurses' navigational role that one medical student and one nephrology fellow that were at the clinic at the time of our study were directed to shadow the nurses and then, later, the dieticians and the pharmacists to get a sense of how the clinic worked. The director of the clinic explained to the nephrology fellow: "The nurses often know more than we do."

There was a lot of overlap in what the different HCPs discussed with the patients. For instance, both the physicians and the pharmacist discussed medications, and the physicians also discussed some physiological symptoms such as itchiness and sleeplessness the nurses had already touched on. The quotes below show two examples of how diet was discussed with the physician, although a visit with the dietician was scheduled immediately afterward.

Patient: Can I have half a banana?

Physician: Yes, if it's in range.

Patient: Sodium, I've been eating out a lot.

Physician: Sodium in blood has nothing to do with what you eat.

Patient: Oh. 
In general, this overlap reinforced the health message but could also lead to gaps in information as for this diabetic patient:

Patient: I have something [juice] available all the time.

Nurse: Good. Something sweet. Good.

Patient: I keep juice around.

Dietician: You need to follow up with protein, or the blood sugar will fall again.

This gap was likely unintentional on the part of the practitioners but nevertheless could become a source of confusion for patients. At times, HCPs seemed to anticipate this gap and reestablished disciplinary boundaries and roles in their dialogue with patients: "I'm out of my depth here, have you already seen the nutritionist?" [physician]; "Make sure you see a social worker next time .... All I can say as a nurse is not to worry" [nurse]. Hence, the HCPs at the clinic maneuvered between multiple roles and relations to one another: The nurses acted as the guiding link between patients and the rest of the clinic staff, and the HCPs often crossed into the areas of expertise of other HCPs, repeated and reinforced one another, and deferred to one another's expertise when necessary.

\section{Outside the clinic walls: the extended health care team}

The care team was not limited to the staff at the clinic. Many other people had a say and a stake in the care of a patient, with differing levels of power and authority in steering the direction of care. It was emphasized at the clinic that all patients have a family physician, and many also see an endocrinologist and/or a cardiologist or other specialists, depending on their care needs. These physicians were not physically at the clinic but were symbolically present through patients' stories and through the patients' medical charts, which contained the diagnoses and treatment decisions made by these physicians. The HCPs at the clinic sometimes praised or validated the work of physicians outside the clinic: "I am really happy that Dr X has followed your blood sugar" [physician]. The nephrologists sometimes used the external physicians as frames of reference or as reinforcement for their own clinical decisions: "The cardiologist says you are doing great!" The patients also sometimes used the nephrologist at the clinic to renegotiate or to get a second opinion on recommendations from external physicians, such as changes in the type of insulin medication.
The HCPs sometimes gave a different advice than what the patients may have received or understood from physicians outside of the clinic:

Wife: Our [general practitioner (GP)] was suspicious of the pancreas because of the urine.

Physician: It's probably the prostate. Probably a misinterpretation. I will check with the GP. You definitely need the prostate examined.

Patient: The drugstore pharmacist said I shouldn't take X and I should take $\mathrm{Y}$ instead.

Physician: Not really. You shouldn't. No need to be on X in my mind. There's no reason to change.

Physician to patient: Potassium is at X. The GP might say it is too high but it is ok.

Nurse: Blood sugar is very good.

Patient: My family doctor wants it lower.

Nurse: (shakes head) It's good.

Hence, there were many medical experts who were "present" in the visits beyond the HCPs at the clinic whose opinions and clinical decisions had a direct bearing on patient care. Their presence was primarily textual, appearing on the pages of patient charts that circulate through the health care system along with the patient.

\section{On the edge of health care: conflicting roles of caregivers}

Another stakeholder in the care of the patient was the caregiver, who was not an official member of the health care team, inside or outside of the clinic, but who nevertheless played an instrumental role during the consultations as well as in the overall care of the patient. Private (paid) caregivers sometimes cooked for the patients and kept records of patients' diet and their blood pressure in addition to accompanying them to medical appointments. Other patients were dependent on support from their children or spouses. These caregivers, both private and family, played an active role in the visits at the clinic. The caregivers asked questions to understand the situation better for themselves ("He is always thirsty. Is that the diabetes?") or to add information that the patient offers to the HCP ("His pee is sour."). At other times, they tried to help the patients express themselves:

Patient: This foot is itchy.

Caregiver: Tingly, after dinner.

They also sought advice from the HCP on how to best care for the patient. One caregiver asked the 
physician: "He eats a lot of olives. He is European, you know? Is that bad?" The physician asked the caregiver to follow up on this query with the dietician. The caregiver spoke to the dietician in the hallway, and the two of them discussed the salt content of olives. The caregiver responded that she would make sure that the patient stops eating them. The patient, who was hard of hearing, was not involved in this conversation. Hence, some caregivers took on a much more directive role in the consultation, to the point where the discussion became more centered around the caregiver rather than the patient:

Pharmacist: How much water do you drink?

Patient: Four glasses a day.

Pharmacist: Are you able to do more?

Caregiver: I'm gonna push him now!

Pharmacist: Yes, even five glasses would be better.

Caregiver (daughter): Her evening meal is now controlled by me. I make it.

Physician to caregiver: You are doing well.

Caregiver: Well, you taught me what to do. I let her have fun and go out with her daughters and have fun in the mornings, but I control the evening meals.

Caregiver (paid professional): He joined a gym in the community center. I am keeping him busy. Don't want him to sit on the couch all day.

Some of the caregivers in our observations also sought and/or were given validation from the HCPs for the good work that they had done in caring for the patients.

Caregiver (daughter): Sounds like the chef gets the [brand name] scarf! (Raises hands up in the air in triumph)

Pharmacist (to caregiver with meticulous records): You guys

keep really good records here!

Hence, we observed a considerable amount of investment of time, effort, decision-making, and emotions on the part of caregivers, who were at times advocates for the patients as a peripheral member of the multidisciplinary care team and who at other times took the attention away from the patient during the consultations.

\section{Discussion}

Key themes in our analysis revolved around boundaries: the professional boundaries between the HCPs in the health care team at the clinic, the boundaries of medical specialties between all the physicians involved in the patient's care, and the boundaries of what constitutes a health care team; namely, the question of the role of the caregiver. Through an exploratory, qualitative descriptive approach, we provide a series of snapshots of a multidisciplinary CKD clinic in situ. There may be limitations in how applicable these findings are to other clinics and chronic illnesses that may have different histories in terms of health care structure specific to the illness, the clinical setting, or the system of funding and administration. However, these vignettes may facilitate HCPs to reflect on MDC approaches in addition to increasing understanding of MDC care in general by functioning as a pilot foundation for future inquiries. The reliability of our findings is strengthened by two researchers conducting the research and member checking.

Scholars in the past have applied Bourdieu's concepts to the field of health and medicine by thinking of health care professions in terms of professional habituses (see Brosnan, ${ }^{21}$ Luke, ${ }^{22}$ Sinclair, ${ }^{23}$ and Rhynas ${ }^{24}$ ). In these studies, each type of HCP brings to the field of CKD care a set of professional priorities, embodied in a sense of clinical know-how and care values. Our observations in the multidisciplinary CKD clinic contribute to this body of work by highlighting instances in which different HCP habituses come to bear on the same patients. The differences between the professional habituses were most evident in the few instances in which the nephrologists provided a different perspective than the patients' family physician or other specialist. These interactions were textual, via patient charts, and thus fall under the type of multidisciplinary care where interactions between HCPs are formal and limited. ${ }^{11}$ In our observations, this formal interaction took place only among physicians inside and outside of the clinic and not among other HCPs inside and outside of the clinic. The level of authority held by each HCP to not only make diagnoses but also record data on the patient's medical chart may determine the degree to which formal multidisciplinary dynamics can take place, a hypothesis that warrants exploration.

In addition to the formal interactions between physicians involved in the care of the patient, the HCPs were brought together to learn across disciplinary boundaries through informal chats between visits in front of the board, as well as during the rounds at the end of each day. The shared documentation tools used in the clinic (the board and the chart) likely facilitated the collaboration and communication in the team ${ }^{11}$ and helped bridge the boundaries as boundary objects. ${ }^{25}$ Through this kind of team structure, the HCPs developed a team disposition within their professional habitus; that is, that it is important and desirable to consult 
with other team members. At the same time, the HCPs may develop specific roles within the team on the basis of their particular professional habitus. For instance, the nurses translated their front-line skills to act as the guiding link between patients and other HCPs at the clinic. The physicians often took on leadership roles by taking control over the board and the rounds. Perhaps this is an area for greater collaborative decision-making that is not fully taken advantage of at the clinic. Another explanation may be that there is an underlying directive philosophy at play in which knowledge is only shared on a need-to-know basis. ${ }^{26}$

The convergence of different professional habituses can have specific consequences for patient care and for patients' understanding of their own health. Although some habituses are readily accepted as part of the team, others are more peripheral. Patients are encouraged to maintain links with family physicians and other specialists. ${ }^{27}$ In our observations at the clinic, the recommendations of these other physicians were sometimes contradicted, and the patients sometimes used these physicians to validate or to reject the nephrologist's advice. We also observed one instance in which different health messages were offered to the patient by two different HCPs within the health care team at the clinic. Although these discrepancies are most likely unintentional, there is a need for clinic staff members to be aware of and reflect on these potential points of contradiction and the effect they may have on patient's understanding of their health and, ultimately, their health outcome.

Our data confirm existing findings that family members who act as caregivers seek positive and affirming encounters with HCPs and that it is important for these individuals to feel empowered to be a positive force in patients' lives. ${ }^{28}$ Our observations show, however, that the empowerment of caregivers may offset the empowerment of patients. Some caregivers (family members or professionals) assume responsibility for the patient's management to the point at which the patient is not part of the decision-making process. There may be cases in which there is a fine line between patientcentered care and caregiver-centered care, especially in cases where the caregiver is more actively involved than the patient in decision-making. Family members influence the visits in many ways, ${ }^{29}$ and when these family members are also caregivers, there are multiple investments involved, including both emotional and financial input as well as time. In these contexts, family members who are also caregivers are guided by two potentially different habituses: one that relates to the patient as family through emotional obligations of love and support and the other as a caregiver and a member of a health team making efforts to care for the patient by following medical advice. The tensions between two habituses may positively or negatively affect the patient. The complex interplay of these two positions for family members who are also caregivers is an important area for further research. The differences between caregivers who are family members and those who are paid professionals also warrant further study.

\section{Conclusion}

Our qualitative observational study of a single CKD clinic yielded several insights into how an MDC team may work in everyday life. The clinic we observed was structured in a way that encouraged cross-disciplinary interactions in the day-to-day function of the clinic. Hence, professional boundaries were highly dynamic: they overlapped and blurred with one another. Physicians outside the immediate clinic were considered to be valuable members of the care team, but their interactions with the clinic were limited and their input was taken up in diverse ways. Understanding the importance of various professional roles and how they are allocated, either formally as part of care design or organically as clinical routine, may help us understand how multidisciplinary care teams function in real life. Finally, caregivers played a significant role in patient care to the point at which caregivers, rather than the patient, were central to the visit, a finding that requires us to rethink the boundaries of a care team and to consider the implications of the caregiver-patient relationship on care.

In this exploratory study, we focused on the practice of multidisciplinary team care of CKD in the context of the clinic visit. We do not yet know whether the findings we have shared hold true in other context, nor have we explored the effects on patient care. However, we know that the meaning HCPs ascribe to MDC most likely shapes their actions and practices, ${ }^{26}$ and our findings have highlighted questions for further study and reflection: How does different or opposing health information from different HCPs affect patients' understanding of their health? How may HCPs bridge potential gaps and differences in the health information they provide to patients? What is the role of caregivers in patient care, and how do their involvement in decision-making and their sense of empowerment affect those of the patients?

\section{Acknowledgments}

We thank Dr Cindy Patton and Dr Scott Lear for their guidance and input during our fieldwork and our writing process. 
This work was supported by LiVWELL Research Group and the Simon Fraser University Community Trust Endowment Fund (31-788031).

\section{Disclosure}

The authors report no conflicts of interest in this work.

\section{References}

1. Levin A, Hemmelgarn B, Culleton B, et al; Canadian Society of Nephrology. Guidelines for the management of chronic kidney disease. CMAJ. 2008;179(11):1154-1162.

2. Mendelssohn DC, Toffelmire EB, Levin A. Attitudes of Canadian nephrologists toward multidisciplinary team-based CKD clinic care. Am J Kidney Dis. 2006;47(2):277-284.

3. Curtis BM, Ravani P, Malberti F, et al. The short- and long-term impact of multi-disciplinary clinics in addition to standard nephrology care on patient outcomes. Nephrol Dial Transplant. 2005;20(1):147-154.

4. Bayliss EA, Bhardwaja B, Ross C, Beck A, Lanese DM. Multidisciplinary team care may slow the rate of decline in renal function. Clin J Am Soc Nephrol. 2011;6(4):704-710.

5. Weiner DE, Tighiouart H, Amin MG, et al. Chronic kidney disease as a risk factor for cardiovascular disease and all-cause mortality: a pooled analysis of community-based studies. J Am Soc Nephrol. 2004;15(5): 1307-1315.

6. Levin A, Lewis M, Mortiboy P, et al. Multidisciplinary predialysis programs: quantification and limitations of their impact on patient outcomes in two Canadian settings. Am J Kidney Dis. 1997;29(4):533-540.

7. Strand H, Parker D. Effects of multidisciplinary models of care for adult pre-dialysis patients with chronic kidney disease: a systematic review. Int J Evid-Based Healthc. 2012;10(1):53-59.

8. Cheong LHM, Armour CL, Bosnic-Anticevich SZ. Primary health care teams and the patient perspective: a social network analysis. Res Social Adm Pharm. 2013;9(6):741-757.

9. Chan M, Johnson D. Multidisciplinary or multifaceted renal care in early chronic kidney disease. Melbourne: Kidney Health Australia; 2012. Available from: http://www.cari.org.au/CKD/CKD\%20early/ Multdisciplinary_Care_ECKD.pdf. Accessed May 14, 2014.

10. Paradis E, Reeves S. Key trends in interprofessional research: a macrosociological analysis from 1970 to 2010. J Interprof Care. 2013;27(2): 113-122.

11. Sheehan D, Robertson L, Ormond T. Comparison of language used and patterns of communication in interprofessional and multidisciplinary teams. J Interprof Care. 2007;21(1):17-30.
12. D'Amour D, Ferrada-Videla M, San Martin Rodriguez L, Beaulieu MD The conceptual basis for interprofessional collaboration: core concepts and theoretical frameworks. $J$ Interprof Care. 2005;19(S1)(Suppl 1): $116-131$.

13. Reeves S, Rice K, Conn LG, Miller KL, Kenaszchuk C, Zwarenstein M. Interprofessional interaction, negotiation and non-negotiation on general internal medicine wards. J Interprof Care. 2009;23(6):633-645.

14. Bourdieu P. Distinction: A Social Critique of the Judgement of Taste. Cambridge, MA: Harvard University Press; 1984.

15. Bourdieu P. Outline of a Theory of Practice. Cambridge, UK Cambridge University Press; 1977.

16. Bourdieu P. Practical Reason. Stanford, CA: Stanford University Press; 1998.

17. Bourdieu P. The Social Structures of the Economy. Malden, MA: Polity Press; 2005.

18. Seale C. Quality in Qualitative Research. Qual Inq. 1999;5(4): 465-478.

19. Braun V, Clarke V. Using thematic analysis in psychology. Qual Res Psychol. 2006;3(2):77-101.

20. Wahlström R, Dahlgren LO, Tomson G, Diwan VK, Beermann B. Changing Primary Care Doctors' Conceptions - A Qualitative Approach to Evaluating an Intervention. Adv Health Sci Educ Theory Pract. 1997;2(3):221-236.

21. Brosnan C. Making sense of differences between medical schools through Bourdieu's concept of 'field'. Med Educ. 2010;44(7):645-652.

22. Luke H. Medical Education and Sociology of Medical Habitus: It's Not About the Stethoscope! New York: Springer; 2003.

23. Sinclair S. Making Doctors. Oxford: Berg Publishers; 1997.

24. Rhynas SJ. Bourdieu's theory of practice and its potential in nursing research. JAdv Nurs. 2005;50(2):179-186.

25. Akkerman SF, Bakker A. Boundary crossing and boundary objects. Review Educational Res. 2011;81(2):132-169.

26. Audulv A, Asplund K, Norbergh KG. Who's in charge? The role of responsibility attribution in self-management among people with chronic illness. Patient Educ Couns. 2010;81(1):94-100.

27. Beaulieu M, Levin A. Analysis of multidisciplinary care models and interface with primary care in management of chronic kidney disease. Semin Nephrol. 2009;29(5):467-474.

28. Nygårdh A, Wikby K, Malm D, Ahlstrom G. Empowerment in outpatient care for patients with chronic kidney disease - from the family member's perspective. BMC Nurs. 2011;10(21):21.

29. Hasselkus BR. The family caregiver as interpreter in the geriatric medical interview. Med Anthropol Q. 1992;6(3):288-304.
Journal of Multidisciplinary Healthcare

\section{Publish your work in this journal}

The Journal of Multidisciplinary Healthcare is an international, peerreviewed open-access journal that aims to represent and publish research in healthcare areas delivered by practitioners of different disciplines. This includes studies and reviews conducted by multidisciplinary teams as well as research which evaluates the results or conduct of such teams or

\section{Dovepress}

healthcare processes in general. The journal covers a wide range of areas and welcomes submissions from practitioners at all levels, from all over the world. The manuscript management system is completely online and includes a very quick and fair peer-review system. Visit http://www.dovepress.com/testimonials.php to read real quotes from published authors. 